Breinbauer, Ines M.

\title{
Wie man in die Pädagogik einführen und dabei selber viel lernen kann! Bildungswissenschaftliche Grundlagen in der Lehrer*innenbildung für Lehrpersonen mit Fluchthintergrund
}

Kremsner, Gertraud [Hrsg.]; Proyer, Michelle [Hrsg.]; Biewer, Gottfried [Hrsg.]: Inklusion von Lehrkräften nach der Flucht. Über universitäre Ausbildung zum beruflichen Wiedereinstieg. Bad Heilbrunn : Verlag Julius Klinkhardt 2020, S. 95-99

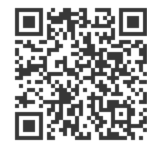

\section{Quellenangabe/ Reference:}

Breinbauer, Ines M.: Wie man in die Pädagogik einführen und dabei selber viel lernen kann! Bildungswissenschaftliche Grundlagen in der Lehrer*innenbildung für Lehrpersonen mit Fluchthintergrund - In: Kremsner, Gertraud [Hrsg.]; Proyer, Michelle [Hrsg.]; Biewer, Gottfried [Hrsg.]: Inklusion von Lehrkräften nach der Flucht. Über universitäre Ausbildung zum beruflichen Wiedereinstieg. Bad Heilbrunn : Verlag Julius Klinkhardt 2020, S. 95-99 - URN: urn:nbn:de:0111-pedocs-189117 - DOI: 10.25656/01:18911

https://nbn-resolving.org/urn:nbn:de:0111-pedocs-189117 https://doi.org/10.25656/01:18911

in Kooperation mit / in cooperation with:

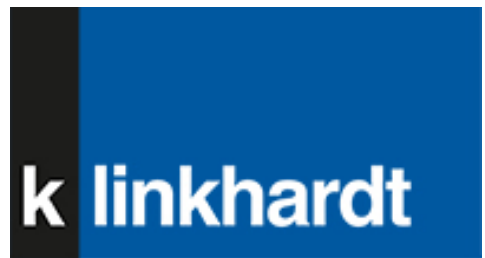

http://www.klinkhardt.de

\section{Nutzungsbedingungen}

Dieses Dokument steht unter folgender Creative Commons-Lizenz: http://creativecommons.org/licenses/by-nc-sa/4.0/deed.de - Sie dürfen das Werk bzw. den Inhalt unter folgenden Bedingungen vervielfältigen, verbreiten und öffentlich zugänglich machen sowie Abwandlungen und Bearbeitungen des Werkes bzw. Inhaltes anfertigen: Sie müssen den Namen des Autors/Rechteinhabers in der von ihm festgelegten Weise nennen. Dieses Werk bzw. der Inhalt darf nicht für kommerzielle Žwecke verwendet werden. Die neu entstandenen Werke bzw. Inhalte dürfen nur unter Verwendung von Lizenzbedingungen weitergegeben werden, die mit denen dieses Lizenzvertrages identisch oder vergleichbar sind.

Mit der Verwendung dieses Dokuments erkennen Sie die Nutzungsbedingungen an.

\section{Terms of use}

This document is published under following Creative Commons-License: http://creativecommons.org/licenses/by-nc-sa/4.0/deed.en - You may copy, distribute and transmit, adapt or exhibit the work in the public and alter, transform or change this work as long as you attribute the work in the manner specified by the author or licensor. You are not allowed to make commercial use of the work. If you alter, transform, or change this work in any way, you may distribute the resulting work only under this or a comparable license.

By using this particular document, you accept the above-stated conditions of use.

\section{Kontakt / Contact:}

peDOcs

DIPF | Leibniz-Institut für Bildungsforschung und Bildungsinformation

Informationszentrum (IZ) Bildung

E-Mail: pedocs@dipf.de

Internet: www.pedocs.de

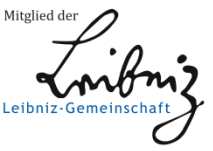




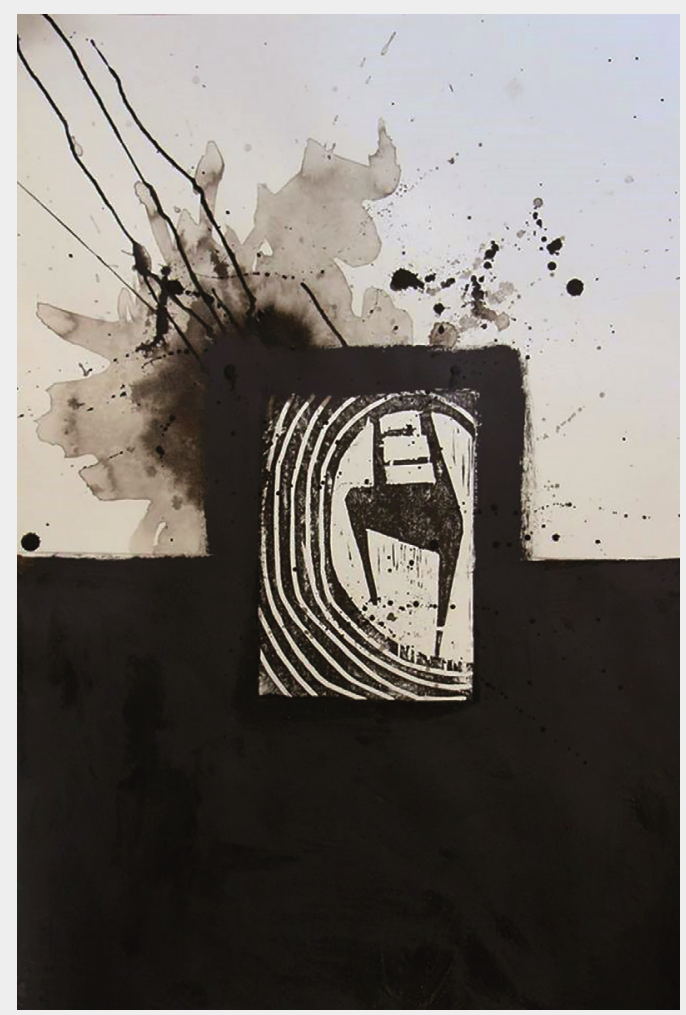

Gertraud Kremsner Michelle Proyer

Gottfried Biewer

(Hrsg.)

Inklusion von Lehrkräften nach der Flucht

Über universitäre Ausbildung zum beruflichen Wiedereinstieg 


\section{Gertraud Kremsner Michelle Proyer Gottfried Biewer (Hrsg.)}

\section{Inklusion von Lehrkräften nach der Flucht}

Über universitäre Ausbildung zum beruflichen Wiedereinstieg 
„Allen Personen gewidmet, die geflïchtet sind, sich derzeit auf der Flucht befinden oder noch flïchten werden. "

Die Publikation des Buches und die Open Access-Veröffentlichung wurde vom Zentrum für Lehrer*innenbildung und vom Postgraduate Center der Universität Wien bezuschusst.

Dieser Titel wurde in das Programm des Verlages mittels eines Peer-Review-Verfahrens aufgenommen. Für weitere Informationen siehe www.klinkhardt.de.

Bibliografische Information der Deutschen Nationalbibliothek Die Deutsche Nationalbibliothek verzeichnet diese Publikation in der Deutschen Nationalbibliografie; detaillierte bibliografische Daten sind im Internet abrufbar über http://dnb.d-nb.de.

2020.ig. (C) by Julius Klinkhardt.

Satz: Tina Obermayr, Wien

Abbildung Umschlagseite 1: Marwa Sarah (Österreich/Syrien) - Black Hole

The painful fact for a refugee or a foreigner is that you will be always looking for a place to belong to, and you will never find it again you will become a foreigner everywhere you go, slowly you will change and do not fit anywhere. and there will always be a black hole. black hole.

Druck und Bindung: AZ Druck und Datentechnik, Kempten.

Printed in Germany 2020.

Gedruckt auf chlorfrei gebleichtem alterungsbeständigem Papier.

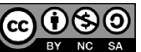

Die Publikation (mit Ausnahme aller Fotos, Grafiken und Abbildungen) ist veröffentlicht unter der Creative Commons-Lizenz: CC BY-NC-SA 4.0 International https://creativecommons.org/licenses/by-nc-sa/4.0/ 


\section{Inhaltsverzeichnis}

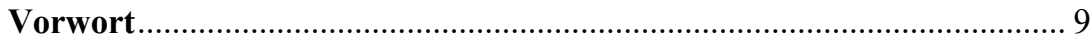

\section{Darstellungen und Forschungen zum Zertifikatskurs}

Gertraud Kremsner, Michelle Proyer und Tina Obermayr

Die Ausgangslage und die Einrichtung des Zertifikatskurses

„Bildungswissenschaftliche Grundlagen für

Lehrkräfte mit Fluchthintergrund“.

Gertraud Kremsner, Michelle Proyer und Alexander Schmölz mit Unterstützung von Helena Deiß, Lisa-Katharina Möhlen, Sarah Hofmann, Marwa Sarah und Tina Obermayr

Das Forschungsprojekt „Qualifizierung von Lehrkräften mit Fluchthintergrund“" 46

\section{Reflexion der Kursinhalte aus Sicht der Lehrenden}

Ines M. Breinbauer

Wie man in die Pädagogik einführen und dabei selber viel lernen kann!

Bildungswissenschaftliche Grundlagen in der Lehrer*innenbildung für Lehrpersonen mit Fluchthintergrund.

Regina Studener-Kuras

„Dass ich Lehrerin bin, das habe ich auf eine recht eigene Art ganz vergessen gehabt!" Lehren und Lernen im Kontext von Flucht und Neubeginn. 100

Michelle Proyer

,Ich male denen die Perspektive“ - Von Praxiserfahrungen zwischen ,bei uns' und ,bei euch', über das Erlernen von ,Reflexion` und hin zu einem ,Ankommen` im österreichischen Schulsystem. 


\section{6 | Inhaltsverzeichnis}

Neda Forghani-Arani

Lived Experience of Teaching Displaced Teachers:

A Postcolonial Reading of Positions, Voices and Representations

Gottfried Biewer

„Inklusive Pädagogik und Vielfalt"

für Lehrkräfte mit Fluchthintergrund

Sabine Krause

Schulforschung und Unterrichtspraxis. Bewegungen zwischen

wissenschaftlicher Abstraktion und „Praxisrelevanz“

Raphael Zahnd und Gertraud Kremsner

Zur vertieften Auseinandersetzung mit Heterogenität in Schulkontexten... 134

Michael Doblmair und Michelle Proyer

Am Ende steht (wieder) die Reflexion

\section{Herausforderungen und Synergien}

Michelle Proyer, Gertraud Kremsner, Gottfried Biewer und Camilla Pellech

Herausforderungen und Synergien aus universitärer Perspektive

Linda Kreuter, Helena Deiß, Lisa-Katharina Möhlen, Kamal Alyouzbashi, Saad Chatto, Sahar Hashemi, Nizar Mousa, Doha Tahlawi, Ahmed Zeki Al Hamid und Jomard Rasul „Werden Träume wahr?“ - Reflexionen der Kursteilnehmer*innen

Marie-Claire Sowinetz

„Nehmen wir das Gute von uns und das Gute von euch so werden wir alle besser." Ein persönlicher Rückblick

auf den Beginn des Zertifikatskurses für geflüchtete Lehrer*innen 166

Katharina Resch

Vier Strategien zur Entwicklung von universitären

Weiterbildungsprogrammen im Bereich Flucht und Migration 
Renate Faistauer, Thomas Laimer und Nicola Kraml

Beitrag zu einer nachhaltigen Sprachförderung für Lehrende

mit Fluchthintergrund - Synergien in der Ausbildung schaffen

und Empowerment bei den Teilnehmer*innen ermöglichen.

Karoline Gerwisch, Denise Strehn, Nicolas Kieffer

und Michelle Proyer

Reflexion der Kurspraktika -

Perspektiven der Mentor*innen und Mentees 184

\section{Internationale Perspektiven}

Annika Käck

Migrant teachers in Swedish teacher education and their

re-entry as professionals

Susanna Malm

Bridging Programmes for Migrant Teachers

and Preschool Teachers in Sweden.

Henrike Terhart, Ariane Elshof und Susanne Preuschoff

Programm für geflüchtete Lehrkräfte an der Universität zu Köln.

Kristina Purrmann, Renate Schüssler, Christina Siebert-Husmann

und Marie Vanderbeke

„Wir haben so lange auf eine Chance gewartet“ -

Potentiale und Herausforderungen des Qualifizierungsprogrammes

Lehrkräfte Plus für geflüchtete Lehrkräfte

Katja Kansteiner, Roswitha Klepser, Sarah Lukas,

Kristin Rheinwald und Tim Kaiser

Integration geflüchteter Lehrkräfte in die Lehrer*innenausbildung in Baden-Württemberg - das IGEL-Programm.

Abschlussbemerkungen 


\section{Wie man in die Pädagogik einführen und dabei selber viel lernen kann! Bildungswissenschaftliche Grundlagen in der Lehrer*innenbildung für Lehrpersonen mit Fluchthintergrund}

Ich stelle im Folgenden meine Erfahrungen mit Modul 1 des Zertifikatskurses in der Form zusammen, dass sowohl mein Verständnis der Aufgabe des Einführens in theoretische Grundlagen der Bildungswissenschaft als auch der Wandel meines Problembewusstseins für diese Vermittlungsaufgabe kenntlich wird.

\section{Du sollst Dir kein Bildnis noch Gleichnis machen - wider die Verbesonderung}

Theoretische Grundlagen der Bildungswissenschaft sind in der Lehrer*innenbildung im Allgemeinen nicht willkommen. Sofern Studierende überhaupt geneigt sind, ,der Pädagogik ${ }^{1}$ einen Stellenwert im Lehramtsstudium zuzubilligen und nicht vor allem die fachlichen und fachdidaktischen Studien für relevant halten, erwarten sie von ,der Pädagogik` eher Ratschläge für in der Schulpraxis zu erwartende Disziplinprobleme, für erfolgreiches Unterrichten, für den Umgang mit Eltern u.ä. und streben nach Erfahrungen in der „Praxis“, die als der eigentliche Lernort angesehen wird (vgl. Schrittesser, Malmberg, Mateus-Berr \& Steger 2014). Nicht sehr viel anders dürfte es den Teilnehmer*innen des Zertifikatskurses ergangen sein. Da sie eigene Unterrichtserfahrung mitbringen, galt das Interesse der besonderen Situation, die sie als Lehrpersonen in Österreich zu erwarten hatten. Zunächst aber mussten sie sich in Modul 1 mit „bildungswissenschaftlichen Grundlagen“ beschäftigen, was ihren Neigungen und Erwartungen anfangs möglicherweise so gar nicht entsprach. Worauf hatte ich mich überhaupt gefasst zu machen? Auf Kolleginnen und Kollegen, denn sie waren in ihren unterschiedlichen Heimatländern wie ich im Lehrberuf tätig. Sie mussten alles hinter sich lassen und sollten nun die Chance für einen neuen Anfang bekommen. Obwohl

1 Die Anführungszeichen markieren das Alltagsbewusstsein bzw. die Alltagssprache. 
- unvermeidlich - schon im Titel des Zertifikatskurses die Zielgruppe als eine besondere angesprochen wird und durch das Kursangebot selber eine Auszeichnung gegenüber anderen Menschen mit Fluchthintergrund erfährt, hatte ich mir vorgenommen, jegliche Verbesonderung $\mathrm{zu}$ vermeiden und Lehre in derselben Weise anzubieten, wie an der Universität sonst auch. Auch bei anderen universitären Lehrveranstaltungen ist Sensibilität für die Aufnahmefähigkeit und -bereitschaft am Platz, aber dennoch können anspruchsvolle Fragen nicht verniedlicht werden, um - wie oftmals gefordert den Intellekt der Studierenden nicht zu strapazieren! Warum sollte ich diese Zielgruppe nicht genau so ernst nehmen? Zudem war ja auch das Curriculum hinsichtlich der Lehrziele ganz in Analogie zu dem „normalen“ Curriculum gestaltet.

\section{Das (bunte) Vorverständnis ist notwendiger Ausgangspunkt der Verständigung}

Es sollte keine Schwierigkeit sein in ,die Pädagogik' einzuführen: Man trifft doch bei jedem Menschen Vorstellungen über Pädagogik an, die er oder sie aus eigener passiver oder aktiver Erziehungserfahrung gewonnen hat! So konnte denn auch Friedrich Schleiermacher an den Beginn seiner Vorlesung aus dem Jahre 1826 den seither viel zitierten und vielfältig interpretierten (vgl. z.B. Schurr 1975) Satz stellen: „Was man im Allgemeinen unter Erziehung versteht, ist als bekannt vorauszusetzen“ (Schleiermacher 1959, 36). An diese Voraussetzung müsste man doch auch bei den Teilnehmer*innen des Zertifikatskurses ansetzen können! Freilich können die damit verbundenen Vorstellungen in die unterschiedlichsten Richtungen gehen! Während die Eine an Kleinkinderziehung denkt, erinnert sich der Andere an erfreuliche oder unerfreuliche Erfahrungen aus der Schulzeit, die Nächste an eine Situation in der Erwachsenenbildung und eine Weitere hat die schon erfahrene oder künftige Lehrer*innenrolle vor Augen. Denkt man die unterschiedlichen Herkunftskulturen dazu, dann sind die verschiedenen Vorerfahrungen und Vorverständnisse von Pädagogik nicht bloß individueller Natur, sondern auch kulturell geprägt, im konkreten Fall durch unterschiedliche Herkunftskulturen von Syrien, Iran, Irak, Tadschikistan bis Tschetschenien. Grundsätzlich ist das Vorverständnis der geradezu notwendige Ausgangspunkt, an dem Verständigungsbemühungen ansetzen müssen. Doch welche Richtung sollen solche Verständigungsbemühungen einschlagen? Sollen sie sich ausschließlich an den Erwartungen der Teilnehmer*innen orientieren und müssen daher zunächst diese ermittelt werden? Besteht die Aufgabe darin, von diesem Vorverständnis her zu einer (wissenschaftlichen) Theorie zu gelangen? Kann 
man diesen unterschiedlichen Zugängen eine allgemeine Basis geben (vgl. Pongratz 2010) und wenn ja, auf welche Weise? Muss man ihnen eine allgemeine Basis geben, - wenn man weiß, dass auch wissenschaftliche Theorien heute plural sind?!

\section{Bloßes Meinen durch die Suche nach Begründungen zu ersetzen ist Aufgabe der Wissenschaft}

Man kann versuchen, den unterschiedlichen Zugängen eine gemeinsame Basis zu geben. Eine solche Suche nötigt jedenfalls zu reflexiver Distanz und erlaubt keine bloße Bestätigung der Vormeinungen: Distanz, in der man des unterschiedlichen Sprachgebrauchs (Sozialisation, Enkulturation, Lernen, Erziehung, Entwicklung, Unterricht, Information, Bildung...) gewahr wird; Irritation, weil man entdeckt, dass man von impliziten oder expliziten normativen Orientierungen ausgeht, die nicht allgemein geteilt werden; Wahrnehmung von Differenzen in den Vorstellungen, mit welchen Maßnahmen diese Ziele erreicht werden können und welche dafür mutmaßlich hinderlich sind. Wenn zu den Ansprüchen an pädagogische Professionalität eine gute Verankerung im theoriefundierten pädagogischen Diskurs und Reflexionsfähigkeit gehören (vgl. Schrittesser 2009), dann ist damit auch die Latte für die Einführung in bildungswissenschaftliche Grundlagen gelegt. Will man nicht bei einem falschen Verständnis von Pluralität stehen bleiben, wonach sich eben jede*r mit einigem Recht ihre*seine pädagogische Wirklichkeit konstruiere, dann kann genau an dieser Stelle ein fruchtbares Nachdenken über ,Allgemeine Bildungswissenschaftliche Grundlagen“ (Universität Wien 2014, 4), wie es im BA-Curriculum für das Lehramtsstudium heißt, beginnen. Das Curriculum fordert genau dies ein, wenn es die Erwartung ausspricht, dass Studierende ,ihre eigene Studien- und Berufsperspektive im Spannungsfeld von persönlichen Absichten, professionellen Herausforderungen und gesellschaftlicher Verantwortung wissenschaftlich fundiert zu reflektieren" (ebd., 7) hätten. Dieser Eintritt in wissenschaftliches Nachdenken über Pädagogik, der zu begrifflicher Klarheit bei der Rede von „pädagogischem Handeln“ auffordert, das Ausweisen der Begründung für Zielvorstellungen einfordert und nach der empirischen Evidenz für die mit pädagogischen Maßnahmen verbundenen Wirkungsannahmen fragt, bleibt unvermeidlich den geschichtlichen, räumlichen und sprachlichen Voraussetzungen der mitgebrachten Auffassungen von Pädagogik verbunden! Unvermeidlich stößt man dabei auf die Frage der Rechtfertigung normativer Erwartungen ebenso wie auf Annahmen über Wirkungen pädagogischer Maßnahmen (vgl. Schäfer 2005). Das gibt Gelegenheit, auch auf die unterschiedlichen forschungsmethodischen An- 
sprüche an die Klärung solcher Fragen in einem späteren Modul zu verweisen. Doch dieser Reflexionsstand gegenwärtiger Bildungswissenschaft kann nicht einfach mitgeteilt werden, auch wenn seitens der Studierenden große Bereitschaft bestünde, jegliche Mitteilung fester Wissensbestände als verbindlich anzunehmen. Weder darf die mitgebrachte Vertrautheit mit bestimmten Vorstellungen von Pädagogik als irrelevant übersprungen werden, noch darf ,das wissenschaftlich begründete Wissen und Urteilen letztlich ein Fremdkörper auf einem dauerhaft maßgeblichen Bodensatz ganz anders gewonnener Grundüberzeugungen“ (Ruhloff 2009, 71) bleiben, noch soll „es unmerklich die Färbung von Selbstverständlichkeit“ annehmen, „die eine Spezifik wissenschaftlichen Wissens entstellt und vergessen macht" (ebd.). „Selbstverständlichkeiten infrage zu stellen und sie durch Begründungszusammenhänge zu ersetzen“ (ebd.) ist der Grundzug von Wissenschaftlichkeit - und die hochschuldidaktische Herausforderung, auch und gerade mit dieser ungewohnten Zielgruppe.

\section{Polylogisches Lehren braucht Zeit}

Es ist nicht schwierig in die Pädagogik einzuführen, wenn man genug Zeit hat, sich auf die Vielfalt von Vorverständnissen genauer einzulassen, ja, ihr zunächst einmal zur Sprache zu verhelfen. Selten zuvor ist mir in der universitären Lehre die Brisanz der temporalen Eigenstruktur von Bildung (vgl. Dörpinghaus 2008) so bewusst geworden. Inkludierende Vorbereitung auf den Lehrberuf in Österreich darf Inklusion nicht so verstehen, als ginge es nur darum, den Stand europäischer bildungswissenschaftlicher Reflexion und österreichischer Schulkultur zu vermitteln, als wäre dies die anzustrebende Norm und als kämen die Studierenden resp. Lehrpersonen nicht ihrerseits aus diversen pädagogischen Traditionen und Schulkulturen. Die Pluralität ist radikaler als zumeist angenommen: Weder kommen alle Teilnehmer*innen aus dem arabischen Raum, noch dürfte dieser als einheitlich verstanden werden; die sprachliche-religiöse-kulturelle Herkunft ist vielfältig. Es ist also soweit unter den gegebenen Rahmenbedingungen möglich - nicht eine traditionsfreie, gar europäisch-überlegene pädagogische Wissenschaft zu vermitteln, auch wenn man möglicherweise bei den ,Integrationswilligen' auf große Bereitschaft stoßen würde, alles anzunehmen, was den erhofften Wiedereinstieg in den Lehrberuf und damit die Wiedererreichung wirtschaftlicher Selbstständigkeit begünstigt. Vielmehr ist eine polylogische Lehre anzustreben, ein echter Austausch in gegenseitiger Anerkennung, als Boden wechselseitiger Horizonterhellung und Horizonterweiterung (vgl. dazu auch Wimmer 2004). Das Aufgreifen dieser Grundidee interkultureller Philosophie begrün- 
det sich nicht im ambivalenten Gedanken der Anerkennung, der die Gefahr in sich trägt, vor allem das bei anderen anzuerkennen, was unseren Erwartungen entspricht und sie ungewollt wieder zu verbesondern. Es leitet sich vielmehr aus der Sorge um blinden Universalismus ab, d.h. naiv eurozentristisches und unhistorisches pädagogisches Denken zu tradieren, muss aber auch einem relativistischen Partikularismus entgegentreten, wonach jede Meinung in irgendeiner Hinsicht berechtigt wäre. Diesen Weg in einer Einführungsvorlesung zu gehen, bedeutet auch, sich auf eine Gratwanderung einzulassen, weil man nicht die Sicherheit vermittelt, die vielfach gerade angehende Lehrpersonen erwarten, die Teilnehmenden aber auch nicht in unbegründeter Selbstsicherheit belassen kann, sondern auf ein Berufsleben mit unabgeschlossenen, ja vielleicht unabschließbaren Problemlösungen einstimmen will. Mag sein, dass das das Sicherheits- und Orientierungsbedürfnis irritiert - aber muss nicht auch Irritation gewagt werden, wenn es um das Anstoßen von Lernprozessen geht (vgl. Meyer-Drawe 2005)?!

\section{Literatur}

Dörpinghaus, A. (2008): Schonräume der Langsamkeit. Grundzüge einer temporalphänomenologischen Erwachsenenpädagogik. In: DIE Zeitschrift für Erwachsenenbildung, 15, H.1, 42-45. Online unter: http://www.diezeitschrift.de/12008/doerpinghaus.htm

[letzter Zugriff: 15.07.2018].

Meyer-Drawe, K. (2005): Anfänge des Lernens. In: Benner, D. (Hrsg.): Erziehung - Bildung Negativität. Weinheim, 24-37 (Zeitschrift für Pädagogik, Beiheft 49).

Pongratz, L. (2010): Sackgassen der Bildung. Pädagogik anders denken. Paderborn/München/ Wien \& Zürich.

Ruhloff, J. (2009): Gefährdende Momente. Vom Einführen in die Pädagogik. In: Kubac, R., Rabl, Ch. \& Sattler, E. (Hrsg.): Weitermachen? Einsätze Theoretischer Erziehungswissenschaft. Würzburg, 70-77.

Schäfer, A. (2005): Einführung in die Erziehungsphilosophie. Weinheim/Basel.

Schleiermacher, F.D.E. (1959): Theorie der Erziehung. Die Vorlesungen aus dem Jahre 1826. In: Ders.: Ausgewählte pädagogische Schriften. Paderborn 1959, 36-243.

Schrittesser, I. (Hrsg.) (2009): Professionalität und Professionalisierung. Einige aktuelle Fragen und Ansätze der universitären Lehrerbildung. Frankfurt/New York \& Wien.

Schrittesser, I., Malmberg, I., Mateus-Berr, R. \& Steger, M. (Hrsg.) (2014): Zauberformel Praxis. Zu den Möglichkeiten und Grenzen von Praxiserfahrungen in der LehrerInnenbildung. Wien.

Schurr, J. (1975): Schleiermachers Theorie der Erziehung. Interpretationen zur Pädagogikvorlesung von 1826. Düsseldorf.

Universität Wien (2014): Allgemeines Curriculum für das Bachelorstudium zur Erlangung eines Lehramts im Bereich der Sekundarstufe (Allgemeinbildung) an der Universität Wien. Mitteilungsblatt Studienjahr 2013/2014 - Ausgegeben am 27.06.2014 - 39. Stück. Wien, 1-14. Online unter: https://www.univie.ac.at/mtbl02/2013_2014/2013_2014_195.pdf [letzter Zugriff: 15.07.2018].

Wimmer, F.M. (2004): Interkulturelle Philosophie. Wien. 